The following corrigenda may be noted. There is no evidence that Leonardo wrote a treatise on the anatomy of the horse: the two volumes of the Feuillets entitled "L'Anatomie du Cheval" contain no anatomy; his dissection of the leg in Quad. V, $22 R$ is his only illustration of the anatomy of the horse (pp. 5 and 35). For "bones" read "horns" and add the figure 3 before "ventricles" (p. 38, par. 5). For "dilation" read "dilatation" (p. 54 f.). Cordæ tendinæ and trabeculæ carnæ are incorrect spellings (p. 63 and Fig. 24 passim). There is no os cordis in the elephant (p. 57). As the figure includes both arteries and veins, the caption should read: "Tree of the Vessels" (pp. 99 and 101). F. J. CoLE

\section{WAVE-GUIDE ENGINEERING}

Waveguide Handbook

Edited by Prof. N. Marcuvitz. (Massachusetts Institute of Technology, Radiation Laboratory Series, No. 10.) Pp. xiv +428. (London: McGrawHill Publishing Co., Ltd., 1951.) 64s.

T $\mathrm{T}$ is now fifty-five years since Lord Rayleigh 1 showed theoretically that it should be possible to propagate two classes of electromagnetic wave inside perfectly conducting tubes of circular or rectangular cross-section; and he directed attention to the important feature that for each class there is a series of characteristic waveforms, with a limiting ('cut-off') frequency for each type, below which no free transmission occurs. It would perhaps appear a matter for some surprise that no further work, neither theoretical nor experimental-- apart from that of Hondros and Debye, Zahn and Schriever on dielectric guides-was carried out for almost forty years after Rayleigh's discovery. The reason for this, however, lies in the fact that wave-guides are scarcely a practical means of transmission at frequencies less than about $1,000 \mathrm{Mc} . / \mathrm{s}$. ; and it was only in the years just before the Second World War that reliable oscillators at sufficiently high frequencies became available, thus allowing the study of wave-guide transmission to be resumed. It was then shown, particularly by workers in the United States, that the attenuation which guided waves would suffer in imperfectly conducting tubes was low enough to make such wave-guides more efficient transmission lines at extremely high frequencies than the familiar parallel-wire or coaxial lines; also, the powerhandling capacity of wave-guides is much superior to that of even the best coaxial line.

The development of high-power centimetre-wave radar systems during the War provided a great stimulus to the investigation of wave-guide circuits and components; such work was indeed a matter of urgency, for without the satisfactory extension of wave-guide techniques it would have been difficult, if not impossible, to realize the full advantages of radar at these very short wave-lengths. A number of books describing the war-time work and subsequent research on the characteristics of wave-guide circuits have already appeared, both in Great Britain and in the United States, but the volume here under review will probably be found more helpful to the radio engineer than any other yet written in view of its essentially practical approach to the subject.

The aim in the "Waveguide Handbook" is to present the salient features in the reformulation of microwave field problems as analogous network problems, and the subject principally treated is the class of electromagnetic boundary-value or diffraction phenomena descriptive of the scattering (or reflexion) properties of discontinuities in wave-guides; the reformulation in this way allows such properties to be calculated in a conventional manner from equivalent microwave networks composed of transmission lines and lumped-constant circuits. During the years 1942-46 a detailed examination of both the field and network aspects of microwave transmission was carried out at the Radiation Laboratory of the Massachusetts Institute of Technology, and J. Schwinger evolved an integral-equation formulation of field problems, the development of which resulted in a rigorous and general theory of microwave structures; and in this treatment conventional low-frequency electrical theory appears as a special case.

Although the main purpose of the book is to present the equivalent-circuit parameters for a large number of wave-guide arrangements-this forms the subject-matter of the later chapters - the first three chapters are devoted to a summary of the theoretical field and network considerations necessary for the derivation and use of the basic transmission-line and equivalent-circuit formalism. Chapter I deals with transmission lines, and Chapter 2 with the characteristics of the different modes of propagation in various kinds of wave-guide; the elements of network theory required for the analysis of the equivalent circuits of $N$-terminal microwave structures are outlined in Chapter 3. Generally, continuous waveguides are used in practice in a manner such that the fields may be described by only a single propagating mode-the dominant mode; in contrast, the complete description of the fields near a discontinuity in the guide requires, in addition to the dominant mode, an infinity of non-propagating modes, but since the effect of the latter modes is restricted to the region of discontinuity, the fields in this region may be regarded as effectively 'lumped'. The equivalent circuits representative of the discontinuities, together with the transmission lines similarly representative of the continuous wave-guide sections, comprise a network which serves to describe the fields almost everywhere within the general wave-guide system.

The remaining chapters, 4-8, contain details of the equivalent-circuit parameters for a variety of $N$-terminal structures, the information being presented both analytically and graphically. In Chapter 4 two-terminal structures such as beyond cut-off and radiative wave-guide terminations are treated, while obstacle and aperture discontinuities in wave-guides, and gratings in free space, are among the four-terminal arrangements discussed in Chapter 5. Chapter 6 deals with six-terminal structures, and contains the equivalent-circuit parameters for a number of $E$ - and $H$-plane $T$ - and $Y$-junctions and bifurcations. Several systems involving eight terminals are described in Chapter 7. Finally, Chapter 8 contains the circuit description of various typical composite microwave structures such as dielectric-filled guides and thick apertures. In these last five chapters there are many tables and graphs containing a wealth of information which will be valuable to the engineer in the design of wave-guide systems.

"Waveguide Handbook" maintains the high standard set by previous volumes of the Radiation Laboratory Series, and it may certainly be recommended as a standard text of reference for all who are in need of guidance for the development of microwave equipment. J. A. SAXToN 